\title{
One-dimensional spherical elementary current systems and their use for determining ionospheric currents from satellite measurements
}

\author{
Liisa Juusola, Olaf Amm, and Ari Viljanen \\ Finnish Meteorological Institute, Space Research Unit, P.O. Box 503, FIN-00101, Finland
}

(Received December 20, 2004; Revised November 18, 2005; Accepted November 19, 2005; Online published May 12, 2006)

\begin{abstract}
The method of 1D spherical elementary current systems (SECS) is a new way for determining ionospheric and field-aligned currents in spherical geometry from magnetic field measurements made by a low-orbit satellite. In contrast to earlier methods, the full ionospheric current distribution, including both divergence-free and curl-free horizontal currents, as well as field-aligned currents, can be determined. Placing infinitely many 2D SECSs of identical amplitudes at a constant latitude results in two types of 1D SECSs, which are independent of longitude, and by superposition can reproduce any ionospheric and field-aligned current system with the same property. One type of the 1D SECSs is divergence-free and toroidal with a poloidal magnetic field, and the other type is curl-free and poloidal. Associated with the divergence of the curl-free type are radial currents. The magnetic field of the combined curl-free 1D SECS and field-aligned currents is toroidal and restricted to the region above the ionosphere. Ionospheric currents are determined by placing several 1D SECSs at different latitudes and choosing their amplitudes in such a way that their combined magnetic field as closely as possible fits the one measured by the satellite. The 1D SECS method has been tested using both modeled and real data from the CHAMP satellite, and found to work excellently in 1D cases.
\end{abstract}

Key words: Ionospheric currents, geomagnetic measurements, spherical elementary current systems.

\section{Introduction}

Magnetic measurements from ground-based magnetometers or satellites can be employed to determine ionospheric electric currents, which are of interest in the ionospheremagnetosphere research. Ground-based measurements by themselves can only yield ionospheric equivalent currents (Fukushima, 1976), whereas measurements above the ionosphere can be used to determine the full current distribution, including horizontal and field-aligned currents (FAC). In addition, a specific advantage of satellite observations over ground-based magnetic measurements is their uniform spatial coverage of the whole Earth. Ground-based stations tend to be irregularly located, leaving large gaps especially on oceans. With additional information on ionospheric electric fields and conductances, also ground-based measurements can yield the full ionospheric current distribution (Untiedt and Baumjohann, 1993) but, unlike magnetic measurements, these are not easily and extensively available.

The technique commonly applied to magnetic satellite data in order to determine ionospheric currents was developed by Olsen (1996). He assumed that only horizontal, not field-aligned currents, contribute to the magnetic field component parallel to the geomagnetic field. The horizontal ionospheric currents were then modeled as a set of infinitely long, straight line currents flowing perpendicular to the satellite orbit plane at a constant height. Limitations of

Copyright (c) The Society of Geomagnetism and Earth, Planetary and Space Sciences (SGEPSS); The Seismological Society of Japan; The Volcanological Society of Japan; The Geodetic Society of Japan; The Japanese Society for Planetary Sciences; TERRAPUB his method are that only one component of the ionospheric current density can be determined, and the geometry is assumed to be partly Cartesian.

Amm (1997) and Amm and Viljanen (1999) introduced the spherical elementary current system (2D SECS) method, which has so far been applied only to determine ionospheric equivalent currents from ground-based measurements (Pulkkinen et al., 2003; Viljanen et al., 2004). Vanhamäki et al. (2003) presented a 1D variant of the method, applicable for determining equivalent currents in electrojet situations, or when data from only one meridian magnetometer chain are available.

In this study, a 1D variant of the 2D SECS method, suitable for determining the full ionospheric current distribution from satellite-based magnetic measurements in spherical geometry, is presented. The goal of this new technique is to calculate simultaneously the zonal, meridional and fieldaligned currents at the altitude of the main ionospheric current flow (here set to $100 \mathrm{~km}$ ). In order to be able to combine the different current components in a meaningful way, all three shall be deduced at the same spatial scale. This scale is limited by the best possible resolution of the zonal currents, which in turn depends on the satellite altitude.

In the following section, the 2D SECS method is shortly reviewed, and in Section 3, the theoretical basis for the new method is laid. This includes deriving the $1 \mathrm{D}$ elementary current systems on which the method is based, and determining application limits of the method. Section 4 deals with testing the method with synthetic ionospheric current distributions, both one- and two-dimensional, and in Section 5 the method is applied to real data from the CHAMP 
satellite. The results of the new method are also compared with those given by the well-established Olsen method. The last section summarises the results and conclusions reached.

\section{The 2D SECS Method}

The method of 2D spherical elementary current systems (2D SECS) was introduced by Amm (1997). It is based on two sets of basis functions in spherical coordinates, which can be used to expand any ionospheric surface current distribution, or any continuously differentiable vector field on a sphere. One of the elementary current systems is divergence-free $\left(J_{d f, 2 D}\right)$, the other one curl-free $\left(J_{c f, 2 D}\right)$. Their definitions in spherical coordinates $\left(r^{\prime}, \theta^{\prime}, \phi^{\prime}\right)$ with unit vectors $\left(\hat{\mathbf{e}}_{r^{\prime}}, \hat{\mathbf{e}}_{\theta^{\prime}}, \hat{\mathbf{e}}_{\phi^{\prime}}\right)$, and with the pole of the elementary system at $\theta^{\prime}=0$, are

$$
\begin{aligned}
& \mathbf{J}_{d f, 2 D}\left(\theta^{\prime}, \phi^{\prime}\right)=\frac{I_{0, d f}}{4 \pi R_{I}} \cot \left(\frac{\theta^{\prime}}{2}\right) \hat{\mathbf{e}}_{\phi^{\prime}} \\
& \mathbf{J}_{c f, 2 D}\left(\theta^{\prime}, \phi^{\prime}\right)=\frac{I_{0, c f}}{4 \pi R_{I}} \cot \left(\frac{\theta^{\prime}}{2}\right) \hat{\mathbf{e}}_{\theta^{\prime}},
\end{aligned}
$$

where $I_{0, d f}$ and $I_{0, c f}$ are the amplitudes of the elementary systems, called scaling factors, and $R_{I}$ is the radius of the ionosphere (Fig. 1).

Associated with the divergence of the curl-free 2D SECS there are field-aligned currents, which are assumed to flow
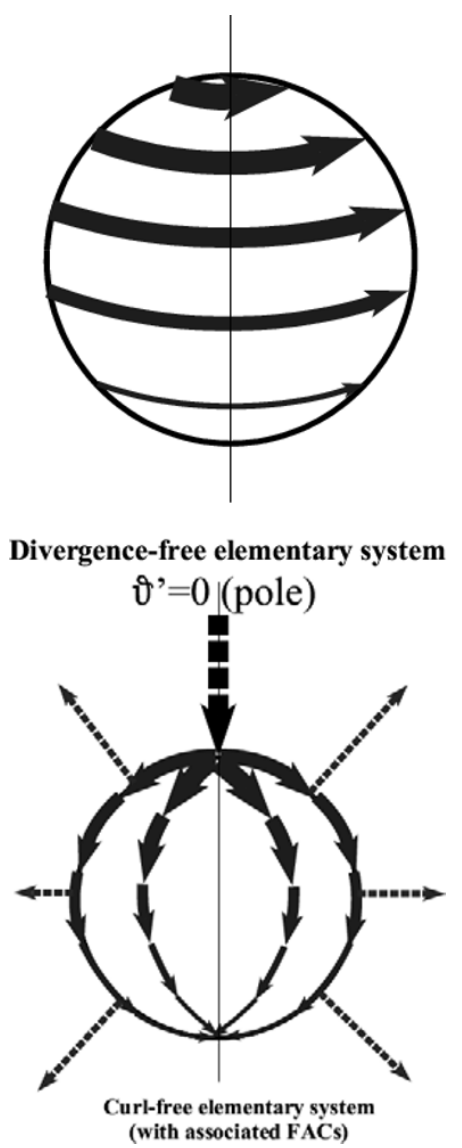

Fig. 1. The divergence-free 2D SECS and the curl-free 2D SECS with associated FACs. radially into or out of the ionosphere

$$
\mathbf{j}_{\|, 2 D}\left(r^{\prime}, \theta^{\prime}, \phi^{\prime}\right)= \begin{cases}\frac{I_{0, c f}}{4 \pi r^{\prime 2}}\left(1-\frac{2}{\sin \theta^{\prime}} \delta\left(\theta^{\prime}\right)\right) \hat{\mathbf{e}}_{r^{\prime}} & , r^{\prime} \geq R_{I} \\ 0 & , r^{\prime}<R_{I}\end{cases}
$$

that is, there is a FAC of strength $I_{0, c f}$ flowing into the ionosphere at the pole, and a uniform density of FACs flowing out of the ionosphere elsewhere, in such a way that the net flow into and out of the ionosphere is zero. Here, currents are denoted by the symbol $I,[I]=\mathrm{A}$, sheet currents by $J$, $[J]=\mathrm{A} / \mathrm{m}$, and current densities by $j,[j]=\mathrm{A} / \mathrm{m}^{2}$.

The magnetic field of the divergence-free 2D SECS was shown by Amm and Viljanen (1999) to have only $B_{r^{\prime}}$ and $B_{\theta^{\prime}}$ components both above and below the ionosphere. The magnetic field of the combined curl-free 2D SECS and FACs, on the other hand, is in the $\phi$ ' direction and confined to the region above the ionosphere for radially flowing FACs (Fukushima, 1976). Thus only the divergence-free 2D SECSs are needed to determine ionospheric equivalent currents.

\section{The 1D SECS Method}

In this context, one-dimensionality means independence of the longitude of the chosen coordinate system. 1D variants of the two types of 2D SECSs can be derived by placing poles of infinitely many 2D SECSs of identical amplitude next to each other at a constant latitude

$$
\mathbf{J}_{\left\{\begin{array}{l}
d f \\
c f
\end{array}\right\}, 1 D}\left(r, \theta, \theta_{0}\right)=\frac{I_{0,\left\{\begin{array}{l}
d f \\
c f
\end{array}\right\}}^{4 \pi R_{I}}}{2 \pi} d \phi_{0} \cot \left(\frac{\theta^{\prime}}{2}\right) \hat{\mathbf{e}}_{\left\{\begin{array}{l}
\phi^{\prime} \\
\theta^{\prime}
\end{array}\right\}},
$$

where $\left(\theta_{0}, \phi_{0}\right)$ are the locations of the 2D SECS poles. The primed coordinates $\left(r^{\prime}, \theta^{\prime}, \phi^{\prime}\right)$ again refer to a spherical coordinate system, where the 2D SECS has its pole at $\theta^{\prime}=0$. The unprimed ones, on the other hand, define the coordinate system where the ionospheric currents are to be determined, and as such could have its pole at the geomagnetic or geographic one, for example.

\subsection{Divergence-free 1D SECS}

Derivation of the divergence-free 1D SECS from Eq. (4) was carried out by Vanhamäki et al. (2003), and the resulting current density is given by

$$
\mathbf{J}_{d f, 1 D}\left(\theta, \theta_{0}\right)=\frac{I_{0, d f}}{2 R_{I}} \hat{\mathbf{e}}_{\phi}\left\{\begin{array}{ll}
-\tan \left(\frac{\theta}{2}\right), & , \theta<\theta_{0} \\
\cot \left(\frac{\theta}{2}\right) & , \theta>\theta_{0}
\end{array} .\right.
$$

The current density is sketched in Fig. 2.

The $\phi$ component of the magnetic field of the system is zero both above and below the ionosphere. The other components can be written using associated Legendre functions $P_{l}^{m}$ (Arfken and Weber, 1995)

$$
\begin{gathered}
B_{r}=\left\{\begin{array}{l}
\frac{\mu_{0} I_{0, d f}}{2 r} \sum_{l=1}^{\infty}\left(\frac{r}{R_{I}}\right)^{l} P_{l}\left(\cos \theta_{0}\right) P_{l}(\cos \theta), r<R_{I} \\
\frac{\mu_{0} I_{0, d f}}{2 r} \sum_{l=1}^{\infty}\left(\frac{R_{I}}{r}\right)^{l+1} P_{l}\left(\cos \theta_{0}\right) P_{l}(\cos \theta), r>R_{I}
\end{array}\right. \\
B_{\theta}=\left\{\begin{array}{l}
\frac{\mu_{0} I_{0, d f}}{2 r} \sum_{l=1}^{\infty}\left(\frac{r}{R_{l}}\right)^{l} \\
\times \frac{1}{l} P_{l}\left(\cos \theta_{0}\right) P_{l}^{1}(\cos \theta), r<R_{I} \\
-\frac{\mu_{0} I_{0, d f}}{2 r} \sum_{l=1}^{\infty}\left(\frac{R_{I}}{r}\right)^{l+1} \\
\times \frac{1^{1}}{l+1} P_{l}\left(\cos \theta_{0}\right) P_{l}^{1}(\cos \theta), r>R_{I}
\end{array}\right.
\end{gathered}
$$




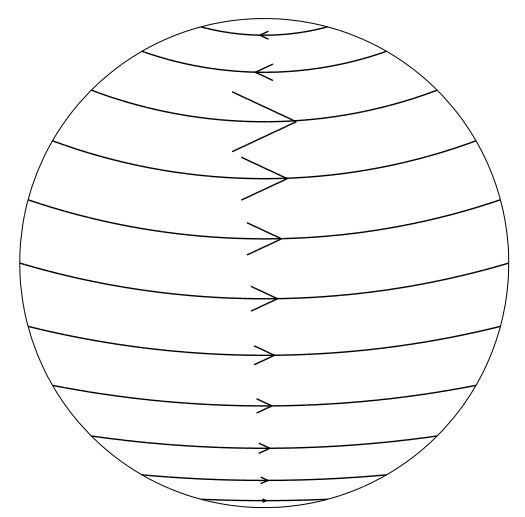

Fig. 2. The divergence-free 1D SECS.

\subsection{Curl-free 1D SECS and FACs}

Calculation of the curl-free 1D SECS closely follows that of the divergence-free one, the details of which were given by Vanhamäki et al. (2003). The result is

$$
\mathbf{J}_{c f, 1 D}\left(\theta, \theta_{0}\right)=\frac{I_{0, c f}}{2 R_{I}} \hat{\mathbf{e}}_{\theta}\left\{\begin{array}{ll}
-\tan \left(\frac{\theta}{2}\right), & \theta<\theta_{0} \\
\cot \left(\frac{\theta}{2}\right) & , \theta>\theta_{0}
\end{array} .\right.
$$

The radial field-aligned currents associated with the curlfree 1D SECS consist again of two parts, FACs flowing uniformly out of the ionosphere, and FACs flowing into the ionosphere at $\theta=\theta_{0}$. The current density of the uniform field-aligned currents at the ionospheric altitude is given by the divergence of the curl-free 1D SECS. The current density of the FACs flowing into the ionosphere at $\theta=\theta_{0}$, on the other hand, is determined by the condition that the net flow into the ionosphere has to vanish. These conditions yield for the FACs

$$
\mathbf{j}_{\|, 1 D}(r, \theta, \phi)=\hat{\mathbf{e}}_{r}\left\{\begin{array}{ll}
\left(\frac{I_{0, c f}}{2 r^{2}}-\frac{I_{0, c f}}{r^{2} \sin \theta_{0}} \delta\left(\theta-\theta_{0}\right)\right) & , r \geq R_{I} \\
0 & , r<R_{I}
\end{array} .\right.
$$

Magnetic field lines of the combined curl-free 1D SECS and its associated FACs are circles around the $z$-axis, and therefore Ampere's law can be applied to calculate their magnetic field. Details of the calculation are given in Appendix A. The result is

$$
\mathbf{B}(r, \theta, \phi)= \begin{cases}-\frac{\mu_{0} I_{0, c f}}{2 r} \cot \left(\frac{\theta}{2}\right) \hat{\mathbf{e}}_{\phi}, r>R_{I}, \theta>\theta_{0} \\ \frac{\mu_{0} I_{0, c f}}{2 r} \tan \left(\frac{\theta}{2}\right) \hat{\mathbf{e}}_{\phi} & , r>R_{I}, \theta<\theta_{0} . \\ 0 & , r<R_{I}\end{cases}
$$

\subsection{Calculation of ionospheric currents}

Measurements of the variation magnetic field components $\left(B_{r}, B_{\theta}\right)$ at locations $\left(r_{i}, \theta_{i}\right), i=1 \ldots n$, can be used to solve the scaling factors $I_{0, d f}$ of the divergence-free 1D SECSs located at $\theta_{0, j}, j=1 \ldots m$, in such a way that their combined magnetic field as closely as possible fits the measured one. Likewise, the measured $B_{\phi}$ component gives the scaling factors $I_{0, c f}$ of the curl-free 1D SECSs. After the scaling factors have been solved, calculating the magnetic field and current density at arbitrary points is a straightforward task.

The problem can be expressed in the matrix form

$$
\mathbf{B}_{\left\{\begin{array}{c}
r, \theta \\
\phi
\end{array}\right\}}=\overline{\bar{M}}_{\left\{\begin{array}{c}
r, \theta \\
\phi
\end{array}\right\}} \cdot \mathbf{I}_{0,\left\{\begin{array}{c}
d f \\
c f
\end{array}\right\}}
$$

where the vector $\mathbf{B}_{r, \theta}$ contains the measured $r$ and $\theta$ components of the magnetic field at locations $\left(r_{i}, \theta_{i}\right)$, the vector $\mathbf{I}_{0, d f}$ scaling factors of the divergence-free 1D SECSs at $\theta_{0, j}$, and the matrix $\overline{\bar{M}}_{r, \theta}$ the effect of the divergence-free $1 \mathrm{D}$ SECSs at $\theta_{0, j}$ on the $r$ and $\theta$ components of the magnetic field at $\left(r_{i}, \theta_{i}\right)$. Likewise, the vector $\mathbf{B}_{\phi}$ contains measurements of the $\phi$ component of the magnetic field at $\left(r_{i}, \theta_{i}\right)$, vector $\mathbf{I}_{0, c f}$ scaling factors of the curl-free 1D SECSs at $\theta_{0, j}$, and the matrix $\overline{\bar{M}}_{\phi}$ the effect of the curl-free 1D SECSs at $\theta_{0, j}$ on the $\phi$ component of the magnetic field at $\left(r_{i}, \theta_{i}\right)$. The scaling factors are obtained by inverting the matrix $\overline{\bar{M}}$

$$
\mathbf{I}_{0,\left\{\begin{array}{c}
d f \\
c f
\end{array}\right\}}=\overline{\bar{M}}_{\left\{\begin{array}{c}
r, \theta \\
\phi
\end{array}\right\}}^{-1} \cdot \mathbf{B}_{\left\{\begin{array}{c}
r, \theta \\
\phi
\end{array}\right\}} .
$$

The number of measurement points is often smaller than the number of 1D SECSs, causing the problem to be underdetermined. Therefore, the matrix inversion has to be performed using a suitable technique, for instance the singular value decomposition (SVD, Press et al., 1992).

In SVD, the matrix $\overline{\bar{M}} \in \mathbf{R}^{n \times m}$ is decomposed into $\overline{\bar{M}}=$ $\overline{\overline{U W V}}^{T}$, where $\overline{\bar{U}} \in \mathbf{R}^{n \times n}$ and $\overline{\bar{V}} \in \mathbf{R}^{m \times m}$ are orthogonal matrices and $\overline{\bar{W}} \in \mathbf{R}^{n \times m}$ a diagonal matrix. The diagonal elements $w_{k k} \geq 0$ of $\overline{\bar{W}}$ are called the singular values of $\overline{\bar{M}}$. If $m>n$ (underdetermined problem), $m-n$ of the $w_{k k}$ 's are zero. Among the rest of the singular values, those that are very small compared to the largest $w_{k k}$ represent the unstable part of the solution. The problem is solved by setting $1 / w_{k k}=0$, if $w_{k k}<\epsilon \cdot \max \left(w_{k k}\right)$. Here $\epsilon$ is an adjustable parameter. The inverse matrix is then $\overline{\bar{M}}^{-1}=\overline{\bar{V}} \operatorname{diag}\left(1 / w_{k k}\right) \overline{\bar{U}}^{T}$.

In addition to satellite measurements, ground-based measurements (cf. Vanhamäki et al., 2003) can be utilized when determining the divergence-free scaling factors. The curlfree ones, of course, have to be determined solely from measurements above the ionosphere, since the curl-free 1D SECS with FACs does not create a magnetic field below the ionosphere. In addition, due to the assumption of radial FACs, which differ from the more realistic dipole ones, the applicability of the curl-free 1D SECSs was determined by respective tests to be restricted to latitudes higher than approximately $45^{\circ}$. The $1 \mathrm{D}$ assumption breaks down at latitudes higher than approximately $80^{\circ}$, and therefore the method does not work in this region either. This restriction, however, can be circumvented by temporally moving the poles of the 1D SECSs to the equator (see Section 5 for an example). Associated with each curl-free elementary system there is also a certain region, whose magnetic measurements cannot be included in determining the amplitude of that particular elementary system. This region, denoted by $F$ in Fig. 3, is located between the assumed radial FAC sheet of the curl-free 1D SECS and the more realistic dipole one. In this region, magnetic fields of the radial and dipole FAC, which are discontinuous at the FAC sheet, changing their direction, are oppositely directed.

The $F$-regions are located at angles

$$
\theta_{0} \leq \theta \leq \theta_{0}+\Delta \theta\left(r, \theta_{0}\right)
$$




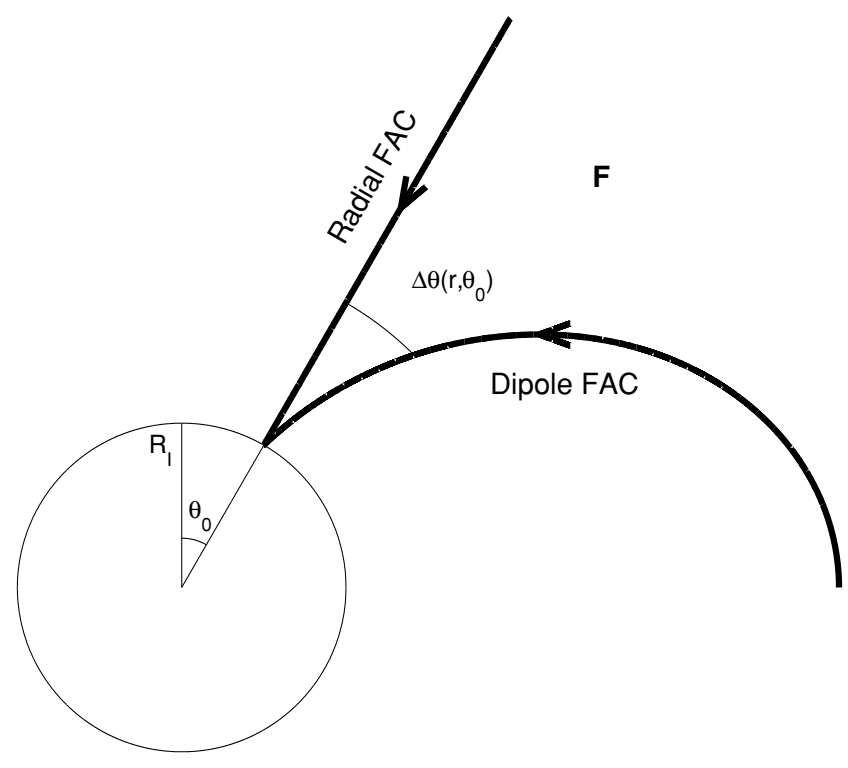

Fig. 3. The $F$-region between the radial FAC sheet of the curl-free 1D SECS and the more realistic dipole FAC sheet. Magnetic measurements in the $F$ region cannot be included in determining the amplitude of the concerned curl-free 1D SECS. The width of the region is denoted by $\Delta \theta\left(r, \theta_{0}\right)$.

and their width, obtained from the geometry of the dipole field line, is

$$
\Delta \theta\left(r, \theta_{0}\right)=\sin ^{-1}\left(\sqrt{\frac{r}{R_{I}}} \sin \theta_{0}\right)-\theta_{0}, R_{I} \leq r \leq \frac{R_{I}}{\sin ^{2} \theta_{0}}
$$

In practise, these regions are left out when determining the curl-free scaling factors by setting the corresponding elements of the matrix $\overline{\bar{M}}_{\phi}$ to zero. This means that $B_{\phi}$ at a certain point $(r, \theta)$ is given by the combined magnetic field of all other 1D SECSs, except those that have the point $(r, \theta)$ located within their $F$-region. Or, reversely, the amplitude of the 1D SECS located at $\theta_{0}$ is determined using only those measurement points that do not fall into the $F$-region of that 1D SECS. An example of the effect of these regions is given in Section 6.

\section{Application Examples-Synthetic Data}

Testing of the 1D SECS method was carried out by applying it to two synthetic current density models: a purely onedimensional electrojet, and a Harang discontinuity model displaying some two-dimensional features.

The starting-point for applying the method to synthetic data was a known ionospheric current density distribution $\left(j_{r}, J_{\theta}, J_{\phi}\right)$. The magnetic field of the current distribution was then calculated along a virtual satellite path, and these 'measured' magnetic field values were used by the 1D SECS method to first determine the scaling factors and from them to recalculate the magnetic field and current distribution. These were then compared with the original magnetic field and current density to determine the competence of the method. The results of the 1D SECS method were also compared with those of the Olsen method.

\subsection{Electrojet}

The electrojet was modeled by a gaussian current density distribution

$$
J_{\phi}=-A e^{-\frac{\left(\theta-\theta_{c}\right)^{2}}{2(\delta \theta)^{2}}}
$$

In order to determine the other current density components, the geomagnetic field was assumed to be $\mathbf{B}=-B \hat{\mathbf{e}}_{r}$, and the convection electric field $\mathbf{E}=E \hat{\mathbf{e}}_{\theta}$, which gave

$$
\left\{\begin{array}{l}
j_{r}=-\nabla \cdot J_{\theta} \hat{\mathbf{e}}_{\theta} \\
J_{\theta}=\Sigma_{P} E \\
J_{\phi}=-\Sigma_{H} E
\end{array},\right.
$$

where $\Sigma_{P}$ and $\Sigma_{H}$ are the Pedersen and Hall conductances. It was assumed that $\alpha=\Sigma_{H} / \Sigma_{P}=2$ (e.g. Untiedt and Baumjohann, 1993), which then yielded the other two current density components in the ionosphere in terms of $J_{\phi}$ :

$$
\begin{gathered}
j_{r}=\frac{1}{R_{I}}\left(\cot \theta-\frac{\theta-\theta_{c}}{(\delta \theta)^{2}}\right) \frac{J_{\phi}}{\alpha} \\
J_{\theta}=-\frac{J_{\phi}}{\alpha} .
\end{gathered}
$$

The magnetic field of the current distribution of the electrojet along a virtual satellite path at $400 \mathrm{~km}$ above the ionosphere was determined by expanding the current density in 1D SECSs.

In order to recalculate the magnetic field and the current density of the electrojet from the magnetic data, the 1D SECSs were placed $1^{\circ}$ apart in the ionosphere. The total number of each type of elementary systems for the electrojet model then became 36. For the Olsen method, the infinitely long, straight line currents were extended from $\theta_{0}=-\pi \ldots \pi$ with a distance corresponding to $1^{\circ}$ between two succesive line currents, and only the $r$ component of the magnetic field was fitted when determing the scaling factors. The results of both methods are given in Fig. 4. Table 1 shows the percentual errors of each fit, defined as

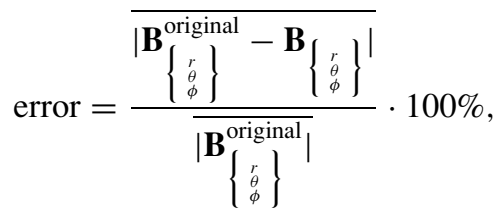

where $\overline{|\mathbf{B}|}$ stands for average of absolute values of the elements of the vector. The error of the current density was likewise determined.

For the 1D SECS method, the errors of the fits are very small, and the curves in Fig. 4 are almost indistinguishable from the original ones. The Olsen method is able to reproduce the electrojet pretty well, but nonetheless the results are not quite as good as those of the 1D SECS method.

Figure 5 illustrates the same electrojet with the satellite passing $2000 \mathrm{~km}$ above the ionosphere. The increased altitude enhances the effects of the geometry, and therefore the partly Cartesian Olsen method cannot perform as well the 1D SECS method. This would be analogous to applying the method to a planet with a radius smaller than that of the Earth. The errors are listed in Table 2. 

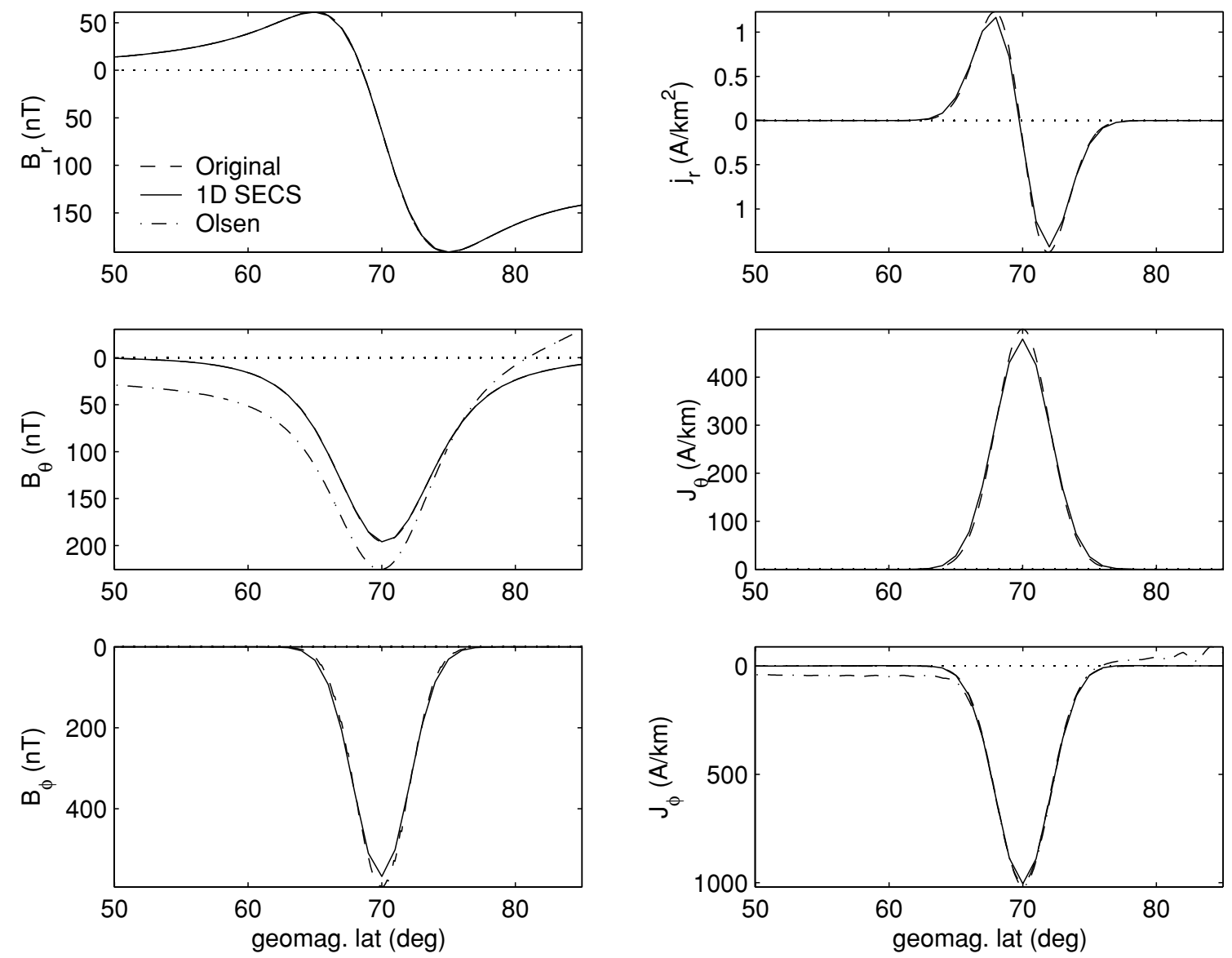

Fig. 4. The three components of the magnetic field and current density of the electrojet model as a function of latitude. $J_{\phi}$ is determined from $B_{r}$ and $B_{\theta}$ by the 1D SECS method, and from $B_{r}$ by the Olsen method. $j_{r}$ and $J_{\theta}$ are calculated from $B_{\phi}$ by the 1D SECS method.

Table 1. Percentual errors of the fits of the electrojet model, displayed in Fig. 4, as given by Eq. (19).

\begin{tabular}{ccccccc}
\hline Method & $B_{r}$ & $B_{\theta}$ & $B_{\phi}$ & $j_{r}$ & $J_{\theta}$ & $J_{\phi}$ \\
\hline 1D SECS & 0.3 & 0.5 & 6 & 8 & 6 & 2 \\
Olsen & $2 \cdot 10^{-4}$ & 50 & - & - & - & 23 \\
\hline
\end{tabular}

Table 2. Percentual errors of the fits of the electrojet model, displayed in Fig. 5, as given by Eq. (19).

\begin{tabular}{ccccccc}
\hline Method & $B_{r}$ & $B_{\theta}$ & $B_{\phi}$ & $j_{r}$ & $J_{\theta}$ & $J_{\phi}$ \\
\hline 1D SECS & 0.06 & 0.05 & 6 & 8 & 6 & 6 \\
Olsen & 0.0012 & 51 & - & - & - & 46 \\
\hline
\end{tabular}

Table 3. Percentual errors of the fits of the Harang discontinuity model, displayed in Fig. 7, as given by Eq. (19).

\begin{tabular}{ccccccc}
\hline Method & $B_{r}$ & $B_{\theta}$ & $B_{\phi}$ & $j_{r}$ & $J_{\theta}$ & $J_{\phi}$ \\
\hline 1D SECS & 1.3 & 98 & 6 & 72 & 65 & 21 \\
Olsen & $3 \cdot 10^{-4}$ & 105 & - & - & - & 22 \\
\hline
\end{tabular}

\subsection{Harang discontinuity}

Figure 6 displays the current density of the Harang discontinuity model, and the path taken by the satellite. The magnetic field of the current distribution was determined using Biot-Savart integration. Figure 7 shows the results of the 1D SECS and Olsen methods applied to the magnetic data of the Harang discontinuity model. The percentual errors of the fits are displayed in Table 3.
This time, the best results for the 1D SECS method were attained by fitting just the $r$ component of the magnetic field when calculating the divergence-free scaling factors. This is most likely due to field-aligned currents, which in a two-dimensional case affect $B_{\theta}$ while leaving $B_{r}$ practically intact. The errors of the $\phi$ component of the magnetic field implicate the success of the fit, whereas errors of the current densities it produces also express the effects due to the two- 

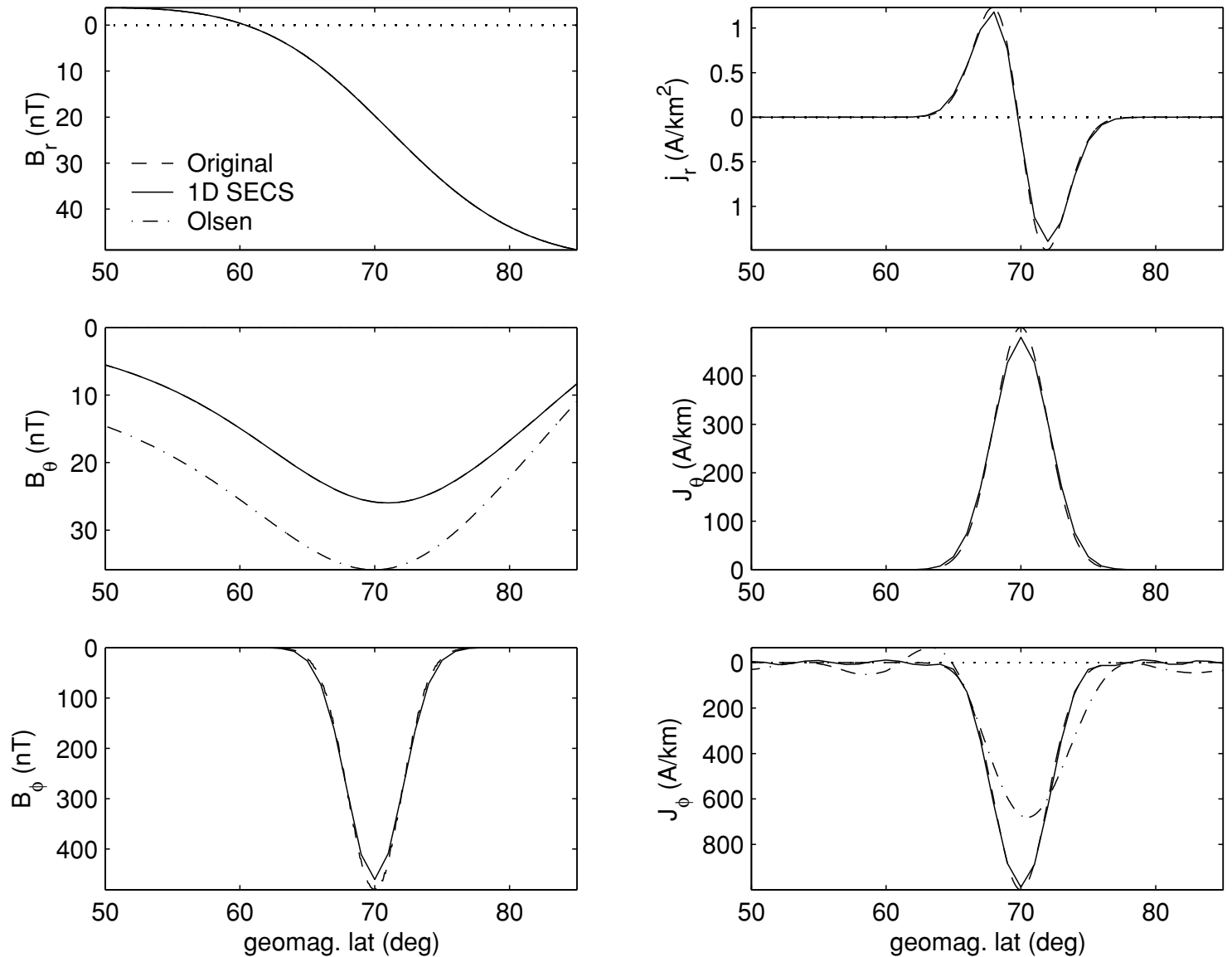

Fig. 5. The same as Fig. 4 but with the satellite passing $2000 \mathrm{~km}$ above the ionosphere instead of $400 \mathrm{~km}$

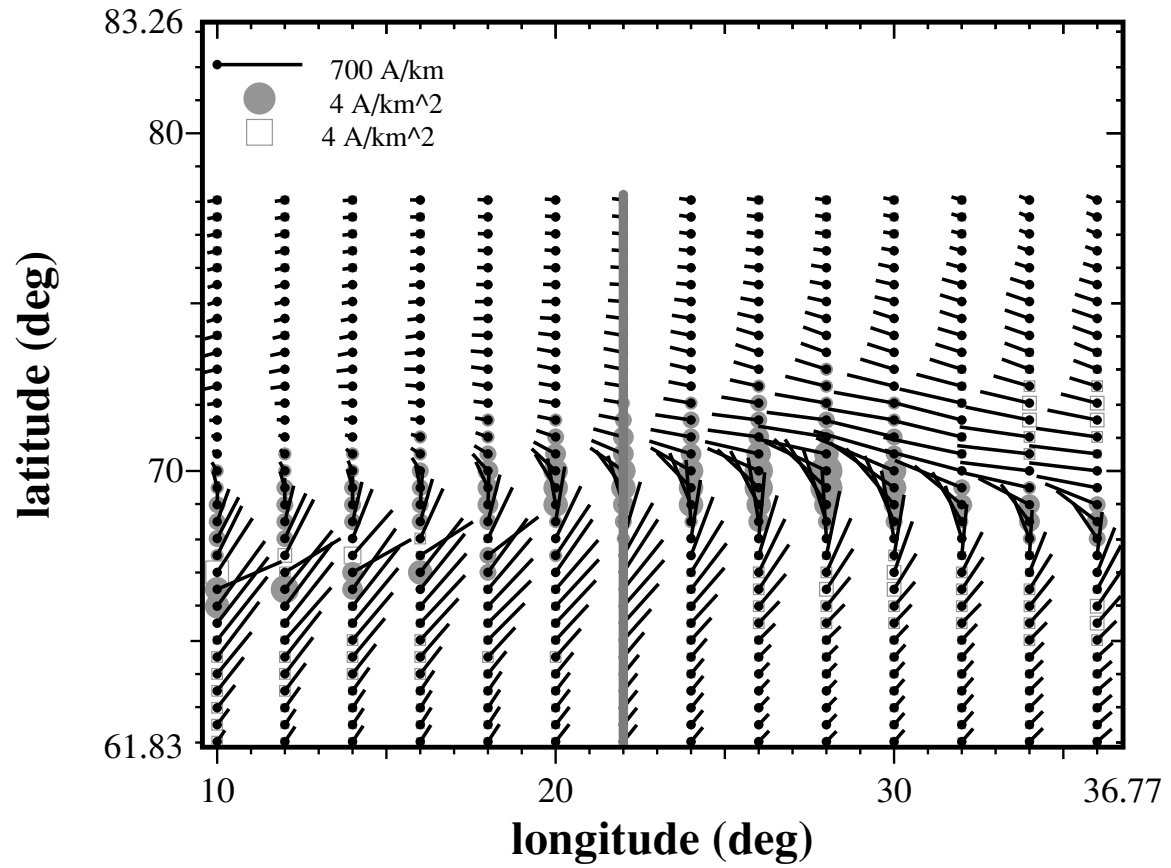

Fig. 6. Current density of the Harang discontinuity model as a function of longitude and latitude. The black vectors display the horizontal current densities, the circles radial upward field-aligned current densities and the squares radial downward field-aligned current densities. The line represents the track of the satellite measuring the magnetic field caused by the current distribution. 

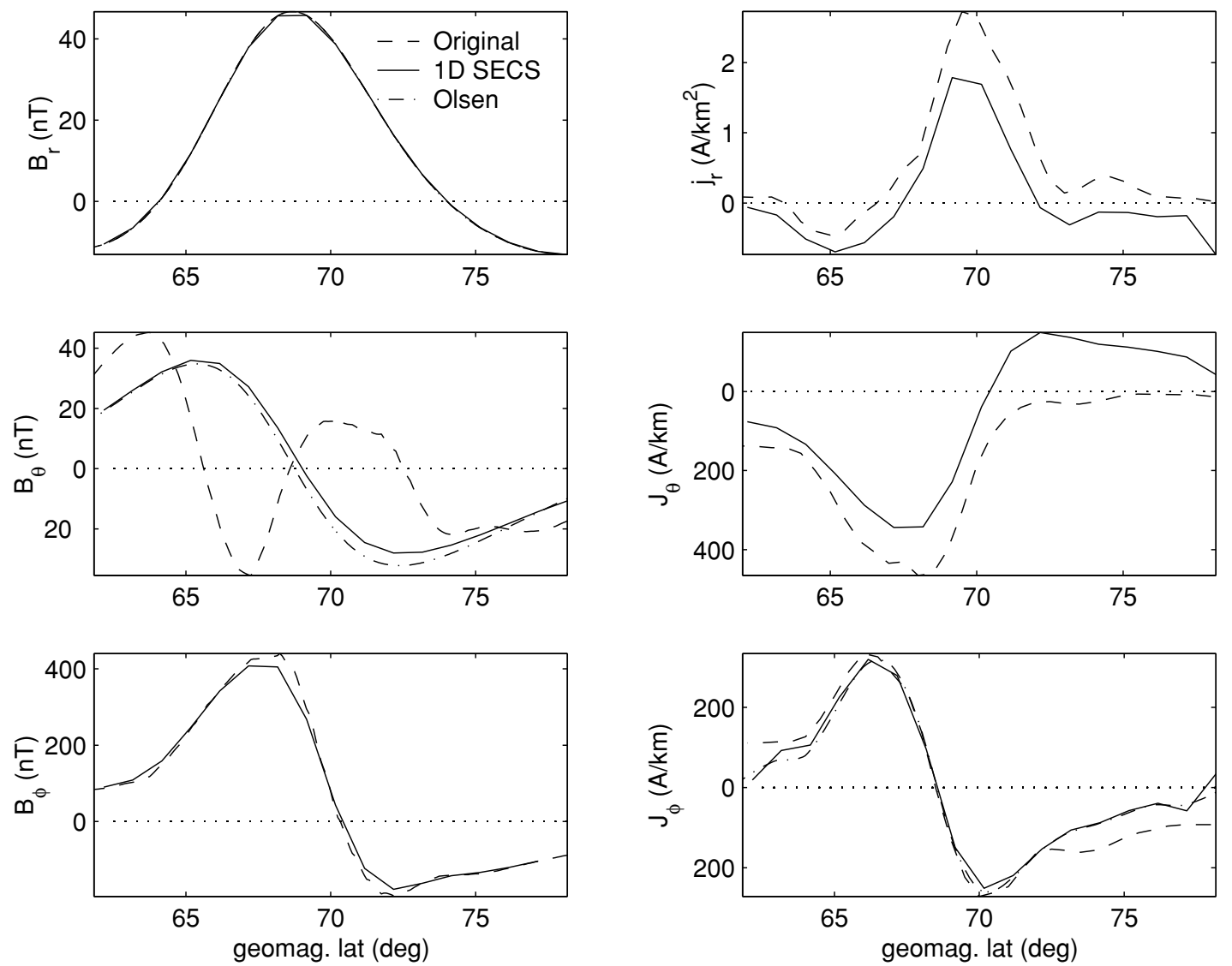

Fig. 7. The three components of the magnetic field and current density of the Harang discontinuity as a function of latitude. $J_{\phi}$ is determined from $B_{r}$. by the 1D SECS method and by the Olsen method. $j_{r}$ and $J_{\theta}$ are calculated from $B_{\phi}$ by the 1D SECS method. The 'original current density' is the current density of the model right below the track of the satellite.

Table 4. Percentual errors of the fits of the CHAMP data, displayed in Fig. 9, as given by Eq. (19).

\begin{tabular}{cccc}
\hline Method & $B_{r}$ & $B_{\theta}$ & $B_{\phi}$ \\
\hline 1D SECS & 6 & 50 & 20 \\
Olsen & 6 & 40 & - \\
\hline
\end{tabular}

dimensionality of the original current distribution. Even $j_{r}$ and $J_{\theta}$ from the excellent $B_{\phi}$ fit do not show an overly good resemblance to the original current distribution along the satellite track.

Though the results for the two-dimensional Harang discontinuity are clearly worse than those for the onedimensional electrojet, the main features of the current density are nonetheless produced pretty well. Performance of both the 1D SECS and Olsen methods appears to be almost equally good.

Further testing with an Omega band model (results not displayed here) revealed that although the performance of the method in a fully two-dimensional case was again worse than with the slightly more one-dimensional Harang discontinuity model, even in such a case the method was able to produce the general shape of the current densities.

\section{Application Example-Real Data}

In order to test the method with real data, a pass of the CHAMP satellite (Ritter et al., 2004) over the IMAGE ground equivalent currents, 06Nov2001 05:05:00

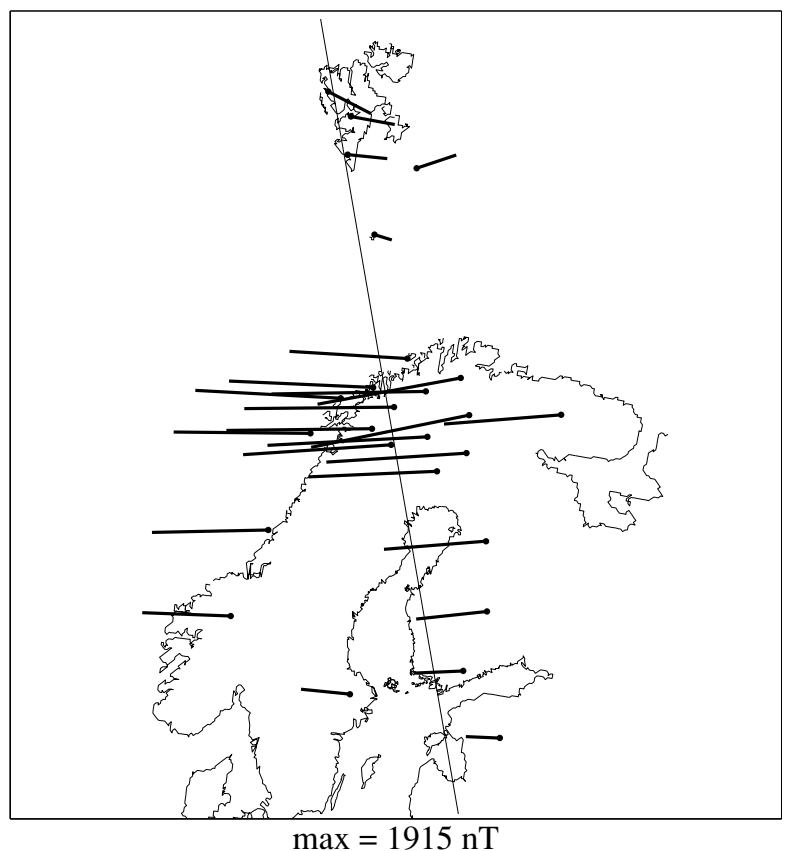

Fig. 8. Equivalent currents, attained by rotating the ground horizontal magnetic field vectors $90^{\circ}$ clockwise, according to the IMAGE magnetometer network on November 6, 2001 at 05:05 UT. The line represents the satellite track. 

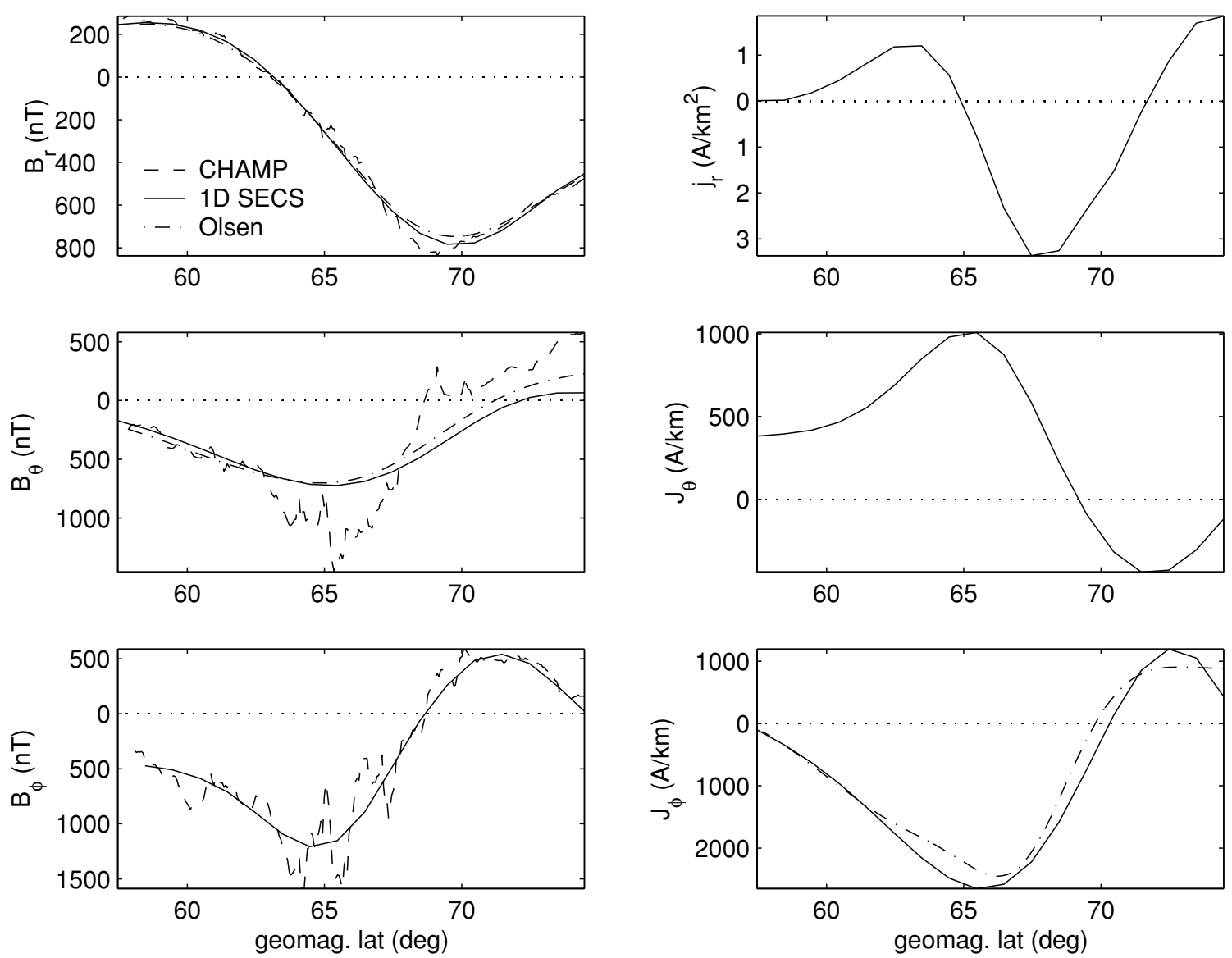

Fig. 9. The three components of the magnetic field and current density measured by the CHAMP satellite on November 6, 2001 at 05:04-05:08 UT, as a function of the geomagnetic dipole latitude. $J_{\phi}$ is determined from $B_{r}$ by the 1D SECS method, and the Olsen method. $j_{r}$ and $J_{\theta}$ are calculated from $B_{\phi}$ by the 1D SECS method.

magnetometer network ${ }^{1}$ on November 6, 2001 at 05:0405:08 UT (8:05-7:26 MLT) was selected. Figure 8 displays a snapshot of the equivalent currents at 05:05 UT. During the overflight, the equivalent current distribution remained pretty much stationary and one-dimensional, as required by the 1D SECS method. When inverting $M_{r, \theta}$ using the SVD, we used $\epsilon=0.01$ and for $M_{\phi}, \epsilon=0.1$.

The magnetic field and current density, as given by the 1D SECS method and the Olsen method, are shown in Fig. 9. In a good agreement with the equivalent currents of Fig. 8, both methods display a westward electrojet centered at $65^{\circ}$ geomagnetic latitude. The oppositely directed currents poleward of the electrojet correspond to the less intense return flow in the polar cap. At $55^{\circ}-65^{\circ}$ and $71^{\circ}-75^{\circ}$ latitudes, the FACs $\left(j_{r}\right)$ are mostly directed away from the ionosphere wheras at $65^{\circ}-71^{\circ}$ they flow into the ionosphere. In general, due to the distance from the ionosphere, both the 1D SECS method and the Olsen method tend to smooth out the small scale fluctuations present in the magnetic data from the satellite. $J_{\theta}$ flows mostly southward on the electrojet proper between the two main regions of the oppositely directed FACs, with some flow northward at latitudes poleward of $70^{\circ}$. Percentual errors of the magnetic field fits are displayed in Table 4. The ground induced currents, which

\footnotetext{
${ }^{1}$ http://www.ava.fmi.fi/image/
}

have been ignored in this analysis, affect mainly the amplitude of $J_{\phi}$ (Olsen, 1996). Finally, Fig. 10 illustrates the application of the 1D SECS method to a polar cap crossing. For that purpose, the $1 \mathrm{D}$ SECS pole was placed at at $0^{\circ}$ geographic latitude and $20^{\circ}$ longitude.

\section{Discussion}

Due to the simple geometry of the magnetic field lines of $J_{\theta}$ and $j_{\|}$in a $1 \mathrm{D}$ case, it might seem that, instead of using 1D SECSs, these currents could be obtained directly by applying Ampere's law, as demonstrated in Appendix B. However, there are two reasons why the 1D SECS method is better. First, in order to be able to compare the meridional and zonal currents meaningfully, the two components have to be calculated at the same scale length. Placing curlfree 1D SECSs at the desired scale length assures that the current system at this (and larger) scale lengths will be optimally matched to the magnetic field data. If Eqs. (B.3) and (B.4) are used directly, unwanted small scale components will be included in the results. Simply averaging the satellite data before using these equations does not produce the desired effect either, since the representation of the averaging operator in the frequency domain is a filter that extends over a wide frequency range. Second, even if one were interested in small scale meridional and field-aligned currents, direct application of Eqs. (B.3) and (B.4) may not necessarily yield the desired quantities at $100 \mathrm{~km}$ altitude. 


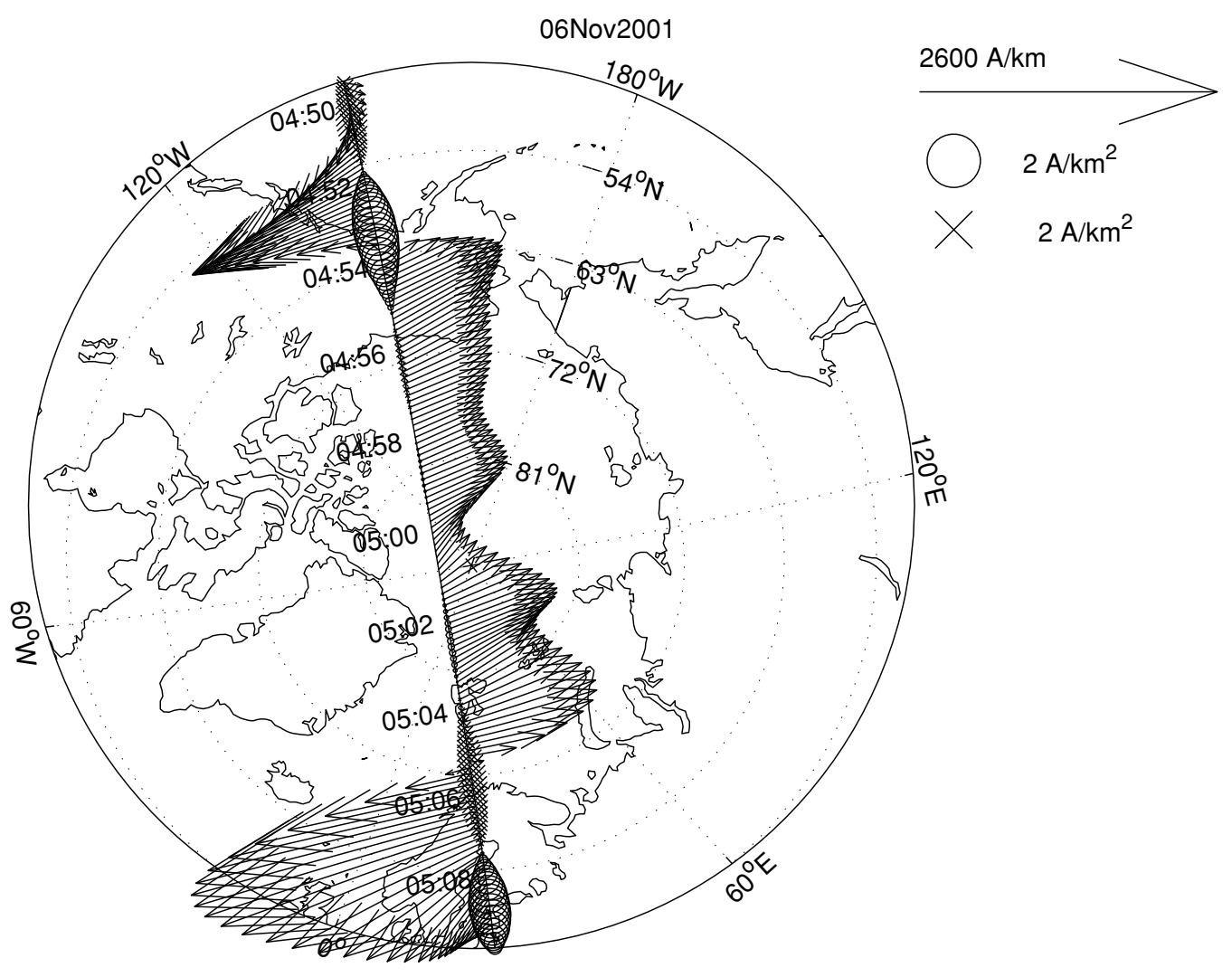

Fig. 10. An example of the application of the 1D SECS method to a polar cap crossing. The horizontal ionospheric currents are denoted by the black arrows, the upward FACs by the circles and the downward FACs by the crosses. In order to compute the currents, the $1 \mathrm{D}$ SECS pole was placed at $0^{\circ}$ latitude and $20^{\circ}$ longitude.

This is due to the fact that the conductive ionosphere causes a damping of the FACs and electric fields of spatial scale smaller than approximately $10-50 \mathrm{~km}$, depending on the ambient conductivities (see e.g. Brekke, 1997, figure 7.35). Hence, if such small scale parts are present in the satellite data, the Ampere's law-approach is not appropriate to this purpose. In the case of quiet events, however, the results of the 1D SECS method and Eqs. (B.3) and (B.4) often become very similar. For comparison, $J_{\theta}$ and $j_{\|}$calculated using the 1D SECS method and Ampere's law are shown on the left hand side of Fig. 11.

\subsection{Effect of the $F$-regions}

The effect of the $F$-regions is also demonstrated in Fig. 11. On the right hand side the matrix elements corresponding to the $F$-regions (Section 3.3) have not been set to zero when determining the curl-free scaling factors, whereas on the left hand side they have. With the satellite flying this low, only approximately $400 \mathrm{~km}$ above the Earth's surface, the effect of not setting the elements to zero is not very significant, as can be seen. $J_{\theta}$ given by the 1D SECS method just appears to have been slightly shifted with respect to the curve derived using Ampere's law. However, with increasing altitude and decreasing latitude the $F$ regions get wider and consequently their effects more pronounced.

\subsection{One-dimensionality}

The 1D SECS method assumes one-dimensionality and therefore gives most reliable results when the current distribution under analysis fulfils this condition. To be able to evalute the quality of the results given by the method, a measure for the one-dimensionality of the situation is needed. One way to achieve this is to use 2D ground-based magnetometer networks, as was illustrated in Section 5. However, these are not always available. Therefore, it is better to be able to do the evaluation relying solely on the magnetic data measured by the satellite. If the divergencefree scaling factors are determined using only the $r$ component of the magnetic field, the deviation of the $\theta$ component of the 1D SECSs' magnetic field from the measured one provides such a measure. The smaller the deviation, the more one-dimensional the current distribution. In a fully 1D case the calculated and measured fields would be equal. In practise, if the shape of the two profiles is approximately the same, the situation appears to be sufficiently 1D. For example, passes of the CHAMP satellite over the IMAGE magnetometer network (like that in Fig. 8) having error of $B_{\theta}$ less than $60 \%$ can be considered to be $1 \mathrm{D}$.

\section{Conclusions}

A new method for determining the full ionospheric current distribution, including horizontal and field-aligned currents, in spherical geometry from low-orbit satellite-based magnetic measurements has been introduced. This 1D SECS method is applicable in cases that are nearly onedimensional and stationary during the satellite overflight. Due to the assumption of radial FACs, the use of the curl-free 1D SECSs is restricted to geomagnetic latitudes higher than approximately $45^{\circ}$. The locations of the 1D 

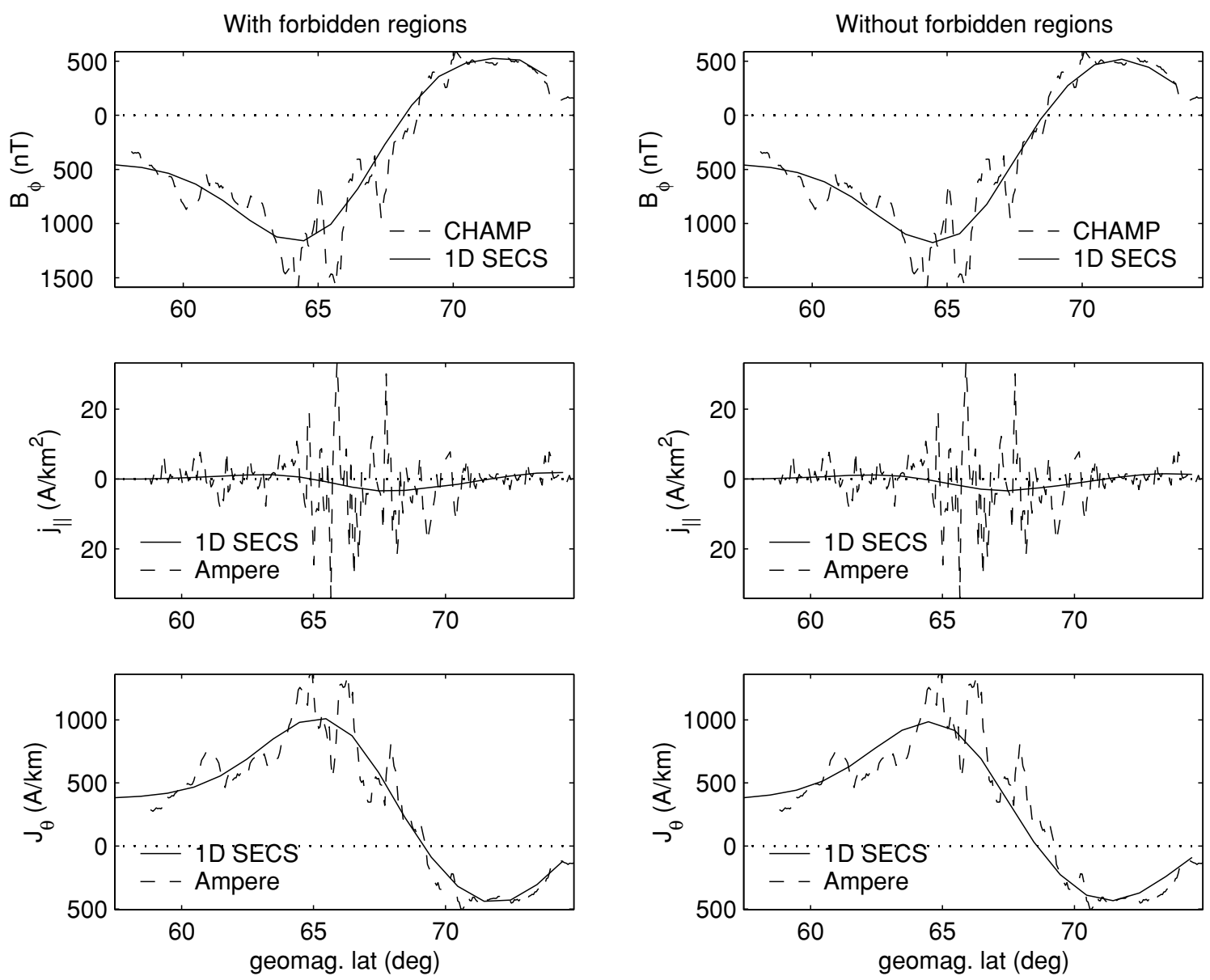

Fig. 11. Left: $j_{\|}$and $J_{\theta}$ calculated from CHAMP data (November 6, 2001, 05:04-05:08 UT) using both the 1D SECS method and directly applying Ampere's law. Right: The same as on the left hand side, except that the matrix elements corresponding to the $F$-regions (Section 3.3) have not been set to zero.

SECSs in the ionosphere can be freely chosen to best accomodate the magnetic field measurements. Only the $r$ and $\theta$ components of the magnetic field are needed to determine the divergence-free horizontal currents, and only the $\phi$ component to calculate the curl-free horizontal currents and field-aligned currents. If ground-based measurements are available, they can be included in determining the divergence-free scaling factors. In cases that are not fully one-dimensional, the ability of the method to determine the divergence-free currents can be improved by fitting just the $r$ component of the magnetic field. The Olsen method, which has commonly been applied to determine ionospheric current from satellite measurements, assumes the geometry to be partly Cartesian, and is only able to produce the part of the horizontal currents perpendicular to the satellite track. The one-dimensionality of the current distribution, and hence the quality of the obtained results can be determined from the difference between the measured $B_{\theta}$ and that of the 1D SECSs' when the amplitudes of the divergence-free 1D SECSs have been determined using only $B_{r}$.

Testing of the 1D SECS method was carried out using both synthetic and real data from the CHAMP satellite. The method was established to work excellently in onedimensional situations, and being able to at least produce the main shape of the currents even if the situation was not strictly one-dimensional. In $1 \mathrm{D}$ cases, performance of the new 1D SECS method appeared to be somewhat better than that of the Olsen method, but in cases which displayed more two-dimensional features, both methods seemed to work almost equally well.

\section{Appendix A.}

In this Appendix, calculation of the magnetic field of the combined curl-free 1D SECS and its associated FACs is outlined.

The magnetic field of the combined curl-free 1D SECS and its associated FACs can be determined employing the vector potential

$$
\mathbf{A}(r, \theta, \phi)=\frac{\mu_{0}}{4 \pi} \int d \mathbf{r}_{1} \frac{\mathbf{J}_{c f, 1 D}\left(r_{1}, \theta_{1}, \phi_{1}\right)}{\left|\mathbf{r}-\mathbf{r}_{1}\right|},
$$

where $\mu_{0}$ is the permeability of the free space, and $\mathbf{J}_{c f, 1 D}$ is given by Eq. (8) (the same for $\mathbf{j}_{\|, 1 D}$, Eq. (9)). Unfortunately, this kind of approach does not easily yield a closed expression for the magnetic field, and therefore is merely used to determine the geometry of the field lines. Once the geometry is known, the magnetic field can be calculated applying Ampere's law in the integral form.

Equation (A.1) for the vector potential can be interpreted as a sum of infinitesimal current density elements placed at $\left(r_{1}, \theta_{1}, \phi_{1}\right)$ and divided by the distance between the cur- 
rent density element and the observation point at $(r, \theta, \phi)$. Due to symmetry in $\phi$ direction, the $\phi=0$ point can be chosen for the observation point without reducing the generality of the analysis. Figure A.12 displays a sketch of the current system. Elements at $\left(r_{1}, \theta_{1}, \phi_{1}\right)$ and $\left(r_{1}, \theta_{1},-\phi_{1}\right)$ have $J_{x}\left(r_{1}, \theta_{1}, \phi_{1}\right)=J_{x}\left(r_{1}, \theta_{1},-\phi_{1}\right), J_{y}\left(r_{1}, \theta_{1}, \phi_{1}\right)=$ $-J_{y}\left(r_{1}, \theta_{1},-\phi_{1}\right), J_{z}\left(r_{1}, \theta_{1}, \phi_{1}\right)=J_{z}\left(r_{1}, \theta_{1},-\phi_{1}\right)$, and their distances from the observation point are equal. The sum of their contributions to the vector potential is therefore a vector with only $x$ and $z$ components. Summing up all the elements pairwise then gives the form of the vector potential at the observation point as $\mathbf{A}(r, \theta, 0)=$ $A_{x}(r, \theta) \hat{\mathbf{e}}_{x}+A_{z}(r, \theta) \hat{\mathbf{e}}_{z}$, which can be written more generally as

$$
\mathbf{A}(r, \theta, \phi)=A_{r}(r, \theta) \hat{\mathbf{e}}_{r}+A_{\theta}(r, \theta) \hat{\mathbf{e}}_{\theta} .
$$

The magnetic field is calculated as $\mathbf{B}=\nabla \times \mathbf{A}$, resulting in

$$
\mathbf{B}=\frac{1}{r}\left(\frac{\partial}{\partial r}\left(r A_{\theta}\right)+\frac{\partial}{\partial \theta} A_{r}\right) \hat{\mathbf{e}}_{\phi},
$$

that is, the magnetic field lines of the combined curl-free 1D SECS and its associated FACs are circles centered on the $z$-axis:

$$
\mathbf{B}(r, \theta, \phi)=B(r, \theta) \hat{\mathbf{e}}_{\phi} .
$$

Ampere's law can now be employed to calculate the magnetic field of the system. The law states that a line integral of the magnetic field $\mathbf{B}$ around a closed path $C$ is equal to $\mu_{0}$ times the total current through a surface $S$ bordered by C

$$
\oint_{C} \mathbf{B} \cdot d \mathbf{l}=\mu_{0} \int_{S} \mathbf{j} \cdot d \mathbf{a} .
$$

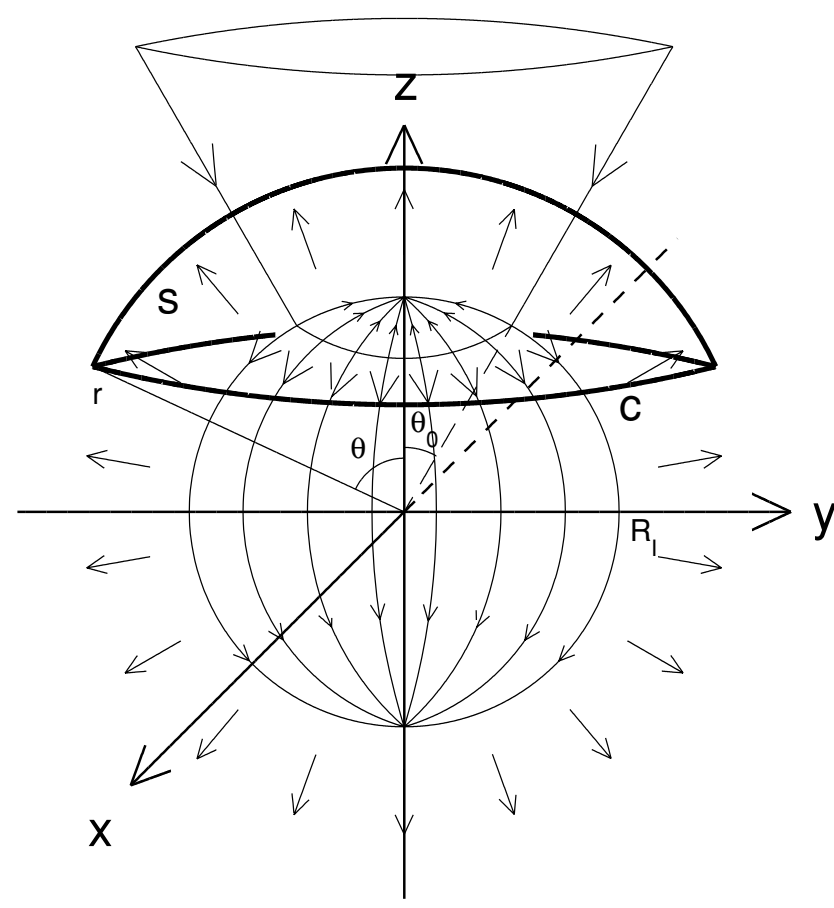

Fig. A.12. The curl-free 1D SECS and associated FACs. Magnetic field of the system can be calculated applying Ampere's law. The concerned surface, bordered by the curve $C$, is denoted by $S . r$ and $\theta$ define the location at which the magnetic field is calculated, and $\theta_{0}$ the angle at which the FAC sheet flows into the ionosphere.

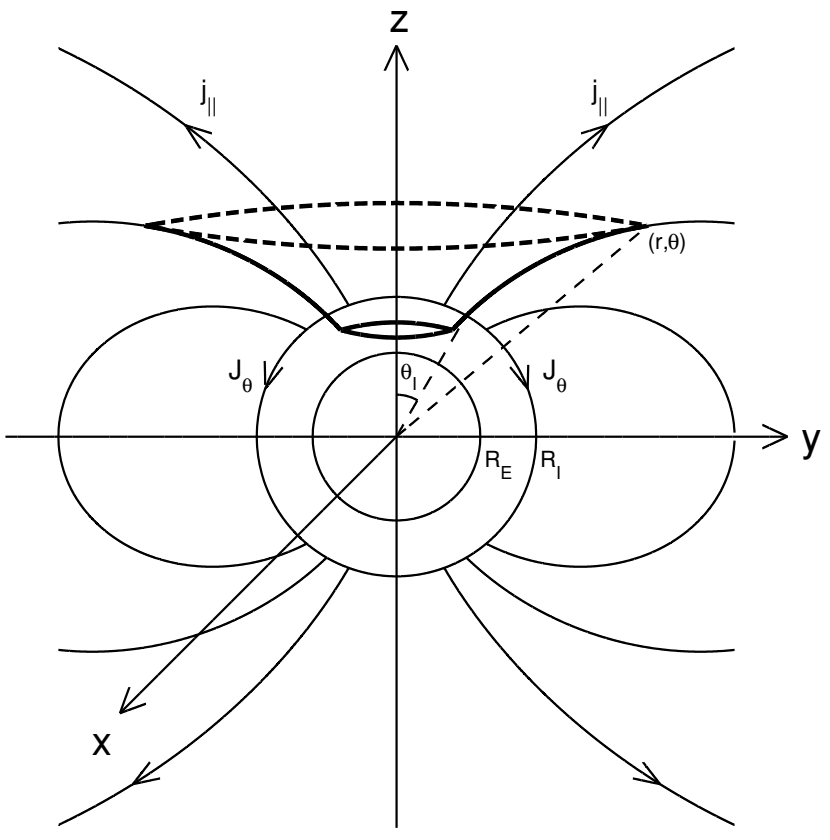

Fig. B.13. The geometry for determining $J_{\theta}$ and $j_{\|}$using Ampere's law. The circular magnetic field line (the thick, slashed line) passing through the point $(r, \theta)$, where the satellite is assumed to lie, borders the funnel-like surface (the thick line), which follows the dipole field and passes through the ionosphere at $\left(R_{I}, \theta_{I}\right)$, closing somewhere below it.

The surface $S$ and curve $C$ are chosen as shown in Fig. A.12. Current passing through the surface $S$ in then solely due to the field-aligned currents, given by Eq. (9). Below the ionosphere $\left(r<R_{I}\right)$, current through the surface is zero, but above $\left(r \geq R_{I}\right)$ it is

$$
\begin{aligned}
\int_{S} \mathbf{j} \cdot \mathbf{a}= & \int_{R_{I}}^{\infty} d r^{\prime} r^{\prime 2} \delta\left(r^{\prime}-r\right) \int_{0}^{\theta} d \theta^{\prime} \sin \theta^{\prime} \\
& \times \int_{0}^{2 \pi} d \phi^{\prime} \frac{I_{0, c f}}{r^{\prime 2}}\left(\frac{1}{2}-\frac{1}{\sin \theta^{\prime}} \delta\left(\theta^{\prime}-\theta_{0}\right)\right) .
\end{aligned}
$$

Performing the integration yields

$$
\int_{S} \mathbf{j} \cdot d \mathbf{a}=\left\{\begin{array}{ll}
-\pi I_{0, c f}(1+\cos \theta) & , r \geq R_{I}, \theta>\theta_{0} \\
\pi I_{0, c f}(1-\cos \theta) & , r \geq R_{I}, \theta<\theta_{0} \\
0 & , r<R_{I}
\end{array},\right.
$$

which combined with Eq. (A.4) and

$$
\oint_{C} \mathbf{B} \cdot d \mathbf{l}=2 \pi r \sin \theta B(r, \theta)
$$

then gives the results of Eq. (10).

\section{Appendix B.}

In this Appendix, $J_{\theta}$ and associated FACs in dipole geometry are calculated directly without using the curl-free 1D SECSs.

Following an argumentation similar to that in Appendix A, the magnetic field lines of $J_{\theta}$ and associated FACs can be determined to be circles centered on the $z$ axis in all 1D cases. The FACs do not need to be radial, but 
actually any kind of geometry fulfilling the $1 \mathrm{D}$ requirement will do just as well. Consequently, $J_{\theta}$ and FACs can be calculated directly using Ampere's law. Figure B.13 shows the respective geometry for FACs flowing along dipole field lines. The circular magnetic field line passing through the point $(r, \theta)$ borders the funnel-like surface, which follows the dipole field and passes through the ionosphere at $\left(R_{I}, \theta_{I}\right)$, closing somewhere below it. Only $J_{\theta}$ contributes to the total current passing through the surface. Therefore, according to Ampere's law,

$$
2 \pi r \sin \theta B_{\phi}(r, \theta)=-\mu_{0} 2 \pi R_{I} \sin \theta_{I} J_{\theta}\left(R_{I}, \theta_{I}\right),
$$

where the angle $\theta_{I}$ is given by

$$
\theta_{I}=\sin ^{-1}\left(\sqrt{\frac{R_{I}}{r}} \sin \theta\right) .
$$

$J_{\theta}$ can now be solved from Eqs (B.1) and (B.2)

$$
J_{\theta}\left(R_{I}, \theta_{I}\right)=-\left(\frac{r}{R_{I}}\right)^{3 / 2} \frac{B_{\phi}(r, \theta)}{\mu_{0}}
$$

and the FACs are given by the current continuity

$$
j_{\|}\left(R_{I}, \theta_{I}\right)=-\frac{1}{r \sin \theta} \frac{\partial}{\partial \theta}\left(\sin \theta J_{\theta}\right) .
$$

These results equal those of the 1D SECS -approach in the case when infinitely many curl-free 1D SECSs are placed in the ionosphere, apart from the geometry of the FACs.

Acknowledgments. The authors wish to thank Dr. Patricia Ritter from GFZ Potsdam for providing the CHAMP satellite data, and Dr. Nils Olsen from Danish Space Research Institute for his valuable advice concerning the application of his method. The two referees are acknowledged for their comments, which greatly improved the outcome.

\section{References}

Amm, O., Ionospheric elementary current systems in spherical coordinates and their application, J. Geomag. Geoelectr., 49, 947-955, 1997.

Amm, O. and A. Viljanen, Ionospheric disturbance magnetic field continuation from the ground to ionosphere using spherical elementary current systems, Earth Planets Space, 51, 431-440, 1999.

Arfken, G. and H. Weber, Mathematical Methods for Physicists, Academic Press, 4th ed., 1995.

Brekke, A., Physics of the Upper Polar Atmosphere, John Wiley \& Sons Ltd, 1997.

Fukushima, N., Generalized theorem for no ground magnetic effect of vertical currents connected with Pedersen currents in the uniformconductivity ionosphere, Rep. Ionos. Space. Res. Japan, 30, 35-40, 1976.

Olsen, N., A new tool for determining ionospheric currents from magnetic satellite data, Geophys. Res. Lett., 23, 3635-3638, 1996.

Press, W. H., B. P. Flannery, S. A. Teukolsky, and W. T. Vetterling, Numerical Recipes, 2nd ed., 973 pp., Cambridge University Press, Cambridge, 1992.

Pulkkinen, A., O. Amm, A. Viljanen, and BEAR Working Group, Ionospheric equivalent current distributions determined with the method of spherical elementary current systems, J. Geophys. Res., 108, 1053, 2003.

Ritter, P., H. Lühr, A. Viljanen, O. Amm, A. Pulkkinen, and I. Sillanpää, Ionospheric currents estimated simultaneously from CHAMP satellite and IMAGE ground based magnetic field measurements: a statistical study at auroral latitudes, Ann. Geophys., 22, 417-430, 2004.

Untiedt, J. and W. Baumjohann, Studies of polar current systems using the IMS Scandinavian magnetometer array, Space Sci. Rev., 63, 245-390, 1993.

Vanhamäki, H., O. Amm, and A. Viljanen, 1-Dimensional upward continuation of the ground magnetic field disturbance using spherical elementary current systems, Earth Planets Space, 55, 613-625, 2003.

Viljanen, A., A. Pulkkinen, O. Amm, R. Pirjola, T. Korja, and BEAR Working Group, Fast computation of the geoelectric field using the method of elementary current systems and planar earth models, Ann. Geophys., 22, 101-113, 2004.

L. Juusola (e-mail: liisa.juusola@fmi.fi), O. Amm, and A. Viljanen 